\title{
Platelet-Derived Growth Factor-B (PDGF-B) Induced by Hypoxia Promotes the Survival of Pulmonary Arterial Endothelial Cells through the PI3K/Akt/Stat3 Pathway
}

\author{
Limin Lia Mengyuan Xu ${ }^{a}$ Xiaoxia Lia Chengfang Lva Xiaoqian Zhanga \\ Hongjuan $\mathrm{Yu}^{\mathrm{a}}$ Mingwen Zhang ${ }^{\mathrm{a}}$ Yueyue Fu ${ }^{\mathrm{a}}$ Hongbin Meng ${ }^{\mathrm{a}}$ Jin Zhou \\ aDepartment of Hematology, the First Affiliated Clinical Hospital of Harbin Medical University, Harbin, \\ People's Republic of China
}

\section{Key Word}

Platelet-derived growth factor-B • Hypoxia • Apoptosis • Pulmonary artery endothelial cells • Stat3

\begin{abstract}
Background/Aims: Pulmonary arterial endothelial plexiform lesions are a basic pathological change associated with pulmonary vascular remodeling and are characterized by the formation of tumorlets as a result of over-growth of endothelial cells. Accumulating evidence suggests that platelet-derived growth factor (PDGF) participates in regulating the progression of pulmonary arterial hypertension. However, whether PDGF promotes the survival of pulmonary arterial endothelial cells (PAECs), as well as the specific molecular mechanisms that underlie its actions, remains unknown. Methods: MTT assays, caspase- 3 and caspase- 9 activity assays and western blot analysis were performed. Results: We found that both the mRNA and protein expression of PDGF-B was induced by hypoxia and that the inhibitory effects exerted by hypoxia on apoptosis were attenuated by inhibitors of PDGF beta. Moreover, PDGF-B inhibited apoptosis in a dose-dependent manner by stimulating the phosphorylation of both Akt and Stat3, and the PI3K/AKT pathway serves as an up-stream participant in the Stat3 activation stimulated by PDGF-B. Additionally, the anti-apoptotic effects of PDGF-B were abolished when PAECs were treated with either an inhibitor or small interfering RNA targeting Stat3. Conclusions: These observations suggest that PDGF-B is induced by hypoxia and protects against apoptosis via the PI3K/Akt/Stat3 signaling pathway.
\end{abstract}

L. Li and M. Xu contributed equally to this work.

Dr. Jin Zhou,

KARGER 125
Department of Hematology, the $1^{\text {st }}$ Affiliated Clinical Hospital of Harbin Medical University, No. 23 Youzheng Street, Nangang District, Harbin, Heilongjiang 150001 (P. R. of China), E-Mail jinzhou81@126.com 
Li et al.: PDGF-B Promotes PAECs Survival via PI3K/Akt/Stat3

\section{Introduction}

Pulmonary arterial hypertension (PAH) is a devastating disease associated with significant morbidity and mortality. This condition is defined as an increase in mean pulmonary artery pressure of at least $25 \mathrm{~mm} \mathrm{Hg}$ at rest and results from an increase in pulmonary vascular resistance due to small vessel remodeling $[1,2]$. Smooth muscle cell hypertrophy, intimal endothelial cell proliferation and the formation of plexiform lesions are the primary histological characteristics of PAH [3]. Endothelial dysfunction occurs in both the pulmonary and systemic circulation and is an important hallmark of PAH [4-6]. Previous studies have demonstrated that pulmonary vascular injury promotes the appearance of apoptosis-resistant pulmonary endothelial cells [4]. Moreover, hypoxia, a key stimulator of $\mathrm{PAH}$, greatly contributes to the development of plexiform lesions, disorganized angiogenesis, and pulmonary vascular remodeling; however, the mechanism for how hypoxia promotes pulmonary intimal hypertrophy remains poorly understood. Therefore, it is necessary to identify the molecules and the corresponding signaling pathways that participate in pulmonary arterial endothelial cell (PAEC) survival under conditions of hypoxia.

The phosphoinositide 3-kinase (PI3K)/Akt pathway is an important endogenous protective mechanism that prevents both cell damage and death, and its activation is crucial for maintaining endothelial cell homeostasis and ensuring endothelial cell survival following vascular injury [7]. Akt activation may lead to cell survival, as well the as the inhibition of apoptosis, via the phosphorylation of Bad, caspase-9, and IкB kinase. STAT3, which is similar to AKT, is a downstream target of various tyrosine kinase receptor signaling pathways and is involved in angiogenesis [8-10]. Most Stat family proteins, including Stat3, exist as monomeric cytosolic proteins; however, upon cytokine stimulation, these proteins are phosphorylated on specific tyrosine residues, form dimers, and subsequently diffuse through the cytosol to a region of nuclear pores before being transported into the nucleus to initiate gene transcription [11-15]. Several reports have focused on the role of Akt phosphorylation and Stat3 translocation and phosphorylation in pulmonary hypertension, although the mechanisms underlying the activation of the PI3K/Akt and Stat3 pathways, as well as whether a link exists between Akt activation and STAT3 activation during the development of PAH, remain unknown.

The up-regulation of PDGF occurs in the setting of PAH, and this phenomenon has been demonstrated previously in the setting of experimental vascular injury. Indeed, PDGF is a pivotal regulator of neointimal enlargement and stimulates vascular smooth muscle cell migration from the media to the neointima [16]. Reportedly, PDGFR- $\beta$, rather than PDGFR- $\alpha$, mediates neointimal expansion in rodents [17]. PDGF-BB, a homodimer of two B subunits, binds to the PDGF $\alpha \alpha-, \alpha \beta-$, or $\beta \beta$-receptor [18]. Following binding and subsequent receptor dimerization, the receptor triggers downstream signaling events, including ERK phosphorylation and transcription factor Sp1 induction [19]. Moreover, PDGF-BB, which is found at high levels in the lung tissues of patients with $\mathrm{PAH}$, is closely related to the development of PAR in patients with PAH [20].

Therefore, we performed several experiments to test the hypothesis that PDGF-B, which is induced by hypoxia, protects against apoptosis via the PI3K/Akt/Stat3 pathway in PAECs. In this study, we sought to confirm that hypoxia induces both the mRNA and protein expression of PDGF-B in PAECs and that the pro-survival effects exerted by hypoxia on PAECs are mediated by PDGF-B. Our findings show that Akt activation serves as an upstream regulator of Stat3, following stimulation by PDGF-B, which protects against mitochondriadependent apoptosis via activation of the PI3K/Akt/Stat3 pathway. Each of these findings demonstrates that PDGF-B is induced by hypoxia and participates in the progression of pulmonary vascular intimal hypertrophy and the development of endothelial plexiform lesions via the PI3K/Akt/Stat3 pathway, which may have important therapeutic implications in the setting of PAH. 
Li et al.: PDGF-B Promotes PAECs Survival via PI3K/Akt/Stat3

\section{Materials and Methods}

\section{Materials}

AG-1296, imatinib, LY294002 and Stattic were purchased from Cayman Chemical Company. Antibodies against Bcl-2, Bax, Akt, p-Akt, Stat3, p-Stat3, cytochrome c, GAPDH and $\beta$-actin were purchased from Cell Signaling Technology. PDGF-B antibody was from Santa Cruz Biotechnology Inc, and Histone H1.2 antibody was bought from Proteintech. Caspase-3 activity kit, Caspase-9 activity kit, MTT and LDH assay kit were obtained from Beyotime Institute of Biotechnology (Haimen, China). All other reagents were from common commercial sources.

\section{Cell preparation and culture}

PAECs were isolated from fresh bovine pulmonary tissues as described previously [21]. These tissues were obtained from a local slaughterhouse in accordance with protocols previously reviewed and approved by the Ethical Committee for Laboratory Animals at Harbin Medical University. The cell types were confirmed by the presence of typical endothelial cell morphology, as well as by positive anti-factor VIII staining. The cells were cultured in DMEM containing $20 \%$ fetal bovine serum (FBS) in a $37^{\circ} \mathrm{C}, 5 \% \mathrm{CO}_{2}$ humidified incubator. Before each experiment, apoptosis was induced via serum deprivation (SD), and the cells were incubated in low-glucose DMEM without serum for $24 \mathrm{~h}$. Passages 2-5 were used for additional experiments.

\section{siRNA design and transfection}

PAECs were transfected with STAT3 small interfering RNAs, which were designed and synthesized by GenePharma. Non-targeted control siRNA (siNC) was used as a negative control. The transfection protocols required that the PAECs were cultured until they reached 30-50\% confluence. A total of $1.5 \mu \mathrm{g}$ of siRNA and $7.5 \mu \mathrm{l}$ of X-treme Gene Transfection Reagent were diluted in serum-free Opti-MEM-1 medium and subsequently mixed together. The mixture was incubated at room temperature for $20 \mathrm{~min}$ and added directly to the cells. Following transfection, the cells were quiesced for $24 \mathrm{~h}$ and then used in experiments.

\section{MTT assay}

PAECs were cultured at a density of approximately $1 \times 10^{4}$ cells per well in 96-well culture plates; the cells were then treated with the above-indicated reagents in low-glucose DMEM without FBS (SD). Ethanol and the other reagents were added at the appropriate concentrations every $24 \mathrm{~h}$. The cells were subsequently incubated in 0.5\% 3-[4, 5-dimethylthiazol-2-yl]-2, 5-diphenyl-tetrazolium bromide (MTT), a yellow mitochondrial dye, is dissolved in sterile PBS buffer for $4 \mathrm{~h}$ at $37^{\circ} \mathrm{C}$. The reaction was terminated by incubating the cells with DMSO for $10 \mathrm{~min}$. The absorbance was measured at $540 \mathrm{~nm}$ using a spectrophotometer, with the amount of blue formazan dye proportional to the number of surviving cells.

\section{LDH assay}

The activity of lactate dehydrogenase (LDH), which released into the culture media, was measured by a cytotoxicity detection kit bought from Beyotime Institute of Biotechnology. The injured cells in cultures were represented by the LDH activities of medium relative to the LDH activity after complete cell lysis, which means total LDH activity, and the total LDH activities were determined using medium containing triton-lysed cellular supernatant. The experiments were carried out as the manufactures' instructions. A portion of culture medium was reacted with an equal volume of LDH substrate solution for $30 \mathrm{~min}$ and then stopped by 5 volume of $0.1 \mathrm{M} \mathrm{NaOH}$, a spectrophotometer was used to measure the absorbance at $440 \mathrm{~nm}$ in sister cultures were treated with $1 / 100$ volume of $10 \%$ triton X-100 and incubated for 30 min at $37^{\circ} \mathrm{C}$.

\section{Western blot analysis}

Proteins from different experimental groups were solubilized and extracted as previously described [22]. The protein concentrations were determined via a bicinchoninic acid protein assay (Pierce, Rockford, IL), where bovine serum albumin (BSA) was used as a standard. Equal amounts of protein (20 $\mu \mathrm{g})$ from each sample were subjected to electrophoresis on an SDS-polyacrylamide gel and transferred onto a nitrocellulose membrane (Millipore, USA). The blots were then incubated in blocking buffer (Tris $20 \mathrm{mM}, \mathrm{pH}$ 7.6, NaCl 150 $\mathrm{mM}$, and Tween $200.1 \%$ ) containing 5\% nonfat dry milk powder for $1 \mathrm{~h}$ at room temperature before being 
Li et al.: PDGF-B Promotes PAECs Survival via PI3K/Akt/Stat3

incubated with the appropriate types and concentrations of antibodies overnight at $4^{\circ} \mathrm{C}$. The following day, the bolts were incubated with horseradish peroxidase-conjugated secondary antibodies and an enhanced chemiluminescence reagent. We used $\beta$-actin as the internal control for all experiments.

\section{Caspase- 3 and caspase- 9 activity assay}

We measured the cleavage of chromogenic caspase substrates, Ac-DEVD-pNA (acetyl-Asp-Glu-Val-Asp p-nitroanilide), which is a caspase-3 substrate, and Ac-LEHD-pNA (acetyl-Leu-Glu-His-Asp p-nitroanilide), a caspase- 9 substrate, as caspase- 3 and caspase- 9 activity, respectively. The experimental procedures were carried on as the manufacture's protocols and previous studies [22, 23]. Almost $50 \mu \mathrm{g}$ of total protein was added to the reaction buffer containing Ac-DEVD-pNA ( $2 \mathrm{mM})$ and Ac-LEHD-pNA (2 mM), and then incubated at $37^{\circ} \mathrm{C}$ for $2 \mathrm{~h}$. The absorbance of yellow pNA cleavaged from its corresponding precursors was measured using a spectrometer at $405 \mathrm{~nm}$. The specific caspase activity, normalized for total proteins of cell lysates, was then expressed as fold of the baseline of control cells cultured in DMEM with 20\% FBS.

Real-time PCR

Applied Biosystems 7300 Fast Real-Time PCR system was used to perform our real-time PCR experiments. Applied Biosystems Primer Express 3.0 was used to design specific primers and a BLAST program was used to confirm the specificity of the primers. Each $20 \mu$ reaction contained 1x SYBR $®$ Premix Ex Taq TM II, $10 \mu \mathrm{M}$ forward and reverse primers, $0.4 \mu \mathrm{l}$ ROX reference dye, and $2 \mu \mathrm{l}$ of cDNA. ABI 7300 Sequence Detector (PerkinElmer Applied Biosystems) was programmed for the PCR conditions: $95^{\circ} \mathrm{C}$ for 30 seconds, 40 cycles of $95^{\circ} \mathrm{C}$ for 5 seconds, and $60^{\circ} \mathrm{C}$ for 30 seconds, and then followed by routine melting curve analysis. The target gene expression relative quantitation (RQ) was calculated by the $2-\Delta \Delta \mathrm{CT}$ method. The first step in the RQ analysis is to normalize target gene expression level to $\beta$-actin $(\Delta \mathrm{Ct}$ ) and the second step is to compare the difference between normalized target gene expressions between different samples $(\Delta \Delta \mathrm{Ct})$. Each experiment was repeated 2-3 times in 3-4 samples.

\section{Statistical analysis}

The composite data were expressed as means \pm SEMs. Statistical analysis was performed with chisquare test, student's t-test or one-way ANOVA followed by Dunnett's test where appropriate. $\mathrm{P}<0.05$ was considered statistically significant.

\section{Results}

Hypoxia induces both the protein and mRNA expression of PDGF-B in PAECS

To determine whether PDGF-B is involved in the hypoxia-mediated growth regulation of PAECs, we first examined whether the expression of PDGF-B is affected by hypoxia. We found that both the protein and the mRNA expression levels of PDGF-B were significantly increased by hypoxia, as determined by western blotting and real-time PCR (Figs. 1A and $1 B, n=3, p<0.05$ ), which suggested that the PDGF pathway was activated under conditions of hypoxia in PAECs.

The inhibitory effects of hypoxia on apoptosis induced by serum deprivation are attenuated by inhibitors of the PDGF-B receptor in PAECs

We next utilized inhibitors of PDGF beta to determine whether the PDGF-B pathway participated in the hypoxia-mediated apoptosis of PAECs. In this study, serum deprivation was used as an apoptotic model. We observed that serum deprivation decreased cell viability and increased the release of lactate dehydrogenase (LDH) in PAECs, an effect that was antagonized by hypoxia. However, the effects of hypoxia on cell viability and cell death were repressed following blockade of the PDGF-B pathway with imatinib and AG-1296 (Figs. $2 \mathrm{~A}$ and $2 \mathrm{~B}, \mathrm{n}=3, \mathrm{p}<0.05$ ). As indicated in Figures $2 \mathrm{C}$ and 2D, hypoxia mitigated the effects exerted by serum deprivation on the activity of both caspase- 3 and caspase- 9 , and the effects exerted by hypoxia were abolished by inhibitors of PDGF beta. These results indicate that PDGF-B mediates the hypoxia-induced survival and growth of PAECs. 
Fig. 1. Both the protein and mRNA expression of PDGF-B is induced by hypoxia in PAECs. A: Hypoxia induced the protein expression of PDGF-B in PAECs. B: The mRNA expression of PDGF-B was up-regulated by hypoxia. All values are denoted as means \pm SEMs from three or more independent batches of cells $\left({ }^{*} \mathrm{p}<0.05\right)$.

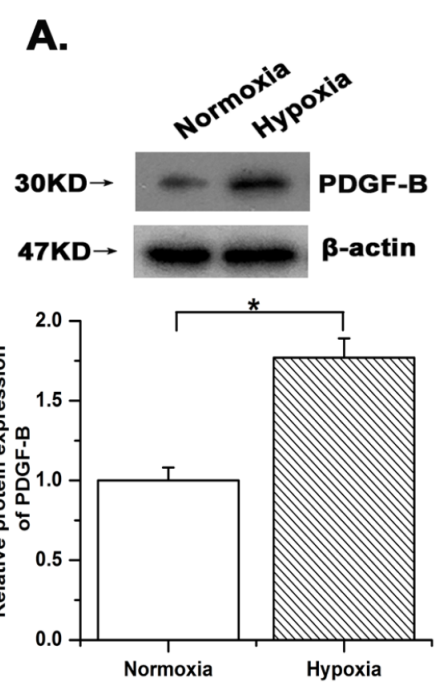

B.

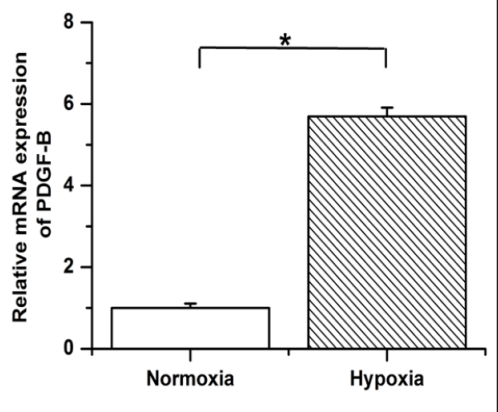

Fig. 2. The inhibitory effects of hypoxia on apoptosis are attenuated by inhibitors of the PDGF-B receptor in PAECs. A: Hypoxia increased cell viability in starved PAECs, and this effect was weakened by both imatinib and AG-1296 (inhibitors of PDGF beta). B: The release of LDH induced by serum deprivation was inhibited by hypoxia in a PDGF-B dependent manner. C: Hypoxia inhibited the activation of caspase-3 in starved PAECs, and this effect was attenuated following blockade of the PDGF-B pathway. D: Hy-

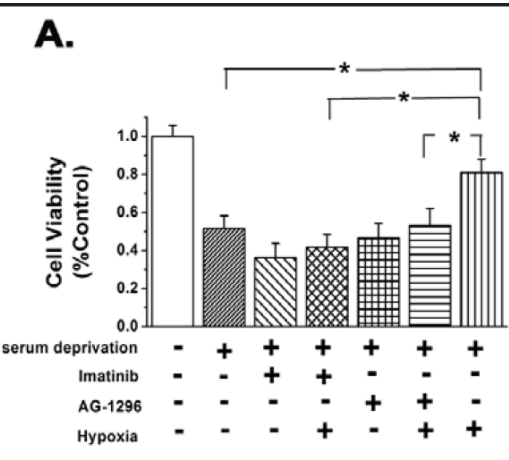

C.

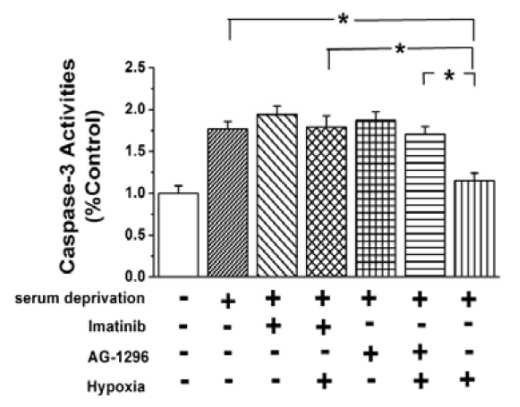

B.

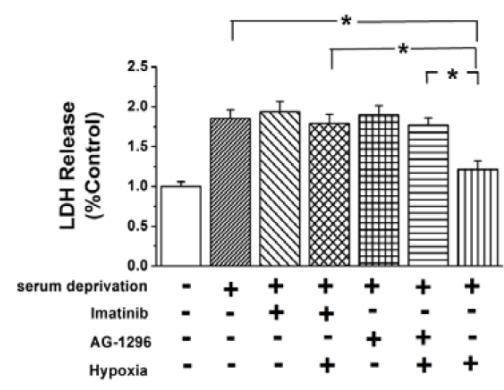

D.

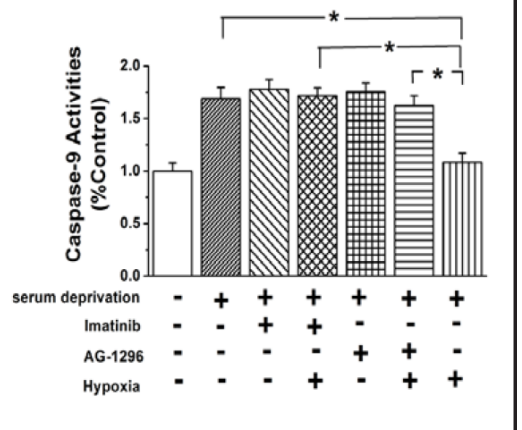

poxia inhibited the activation of caspase- 9 via the PDGF-B pathway. All values are denoted as means \pm SEMs from three or more independent batches of cells $\left({ }^{*} \mathrm{p}<0.05\right)$.

PDGF-B inhibits apoptosis via serum deprivation in a dose-dependent manner

As shown in Figures 3A and 3B, MTT assays and the measurement of caspase-3 activity were performed to analyze the protective effects exerted by different doses of PDGF-B in PAECs following serum deprivation. We found that PDGF-B improved cell viability at a concentration of $10 \mathrm{ng} / \mathrm{ml}$. A similar result was observed for the activity of caspase-3, showing that a concentration of $10 \mathrm{ng} / \mathrm{ml}$ PDGF-B effectively inhibited the activation of caspase- 3 that was induced by serum deprivation in PAECs. These results indicated that PDGF-B, at a concentration of $10 \mathrm{ng} / \mathrm{ml}$, significantly inhibited apoptosis induced by serum deprivation. 


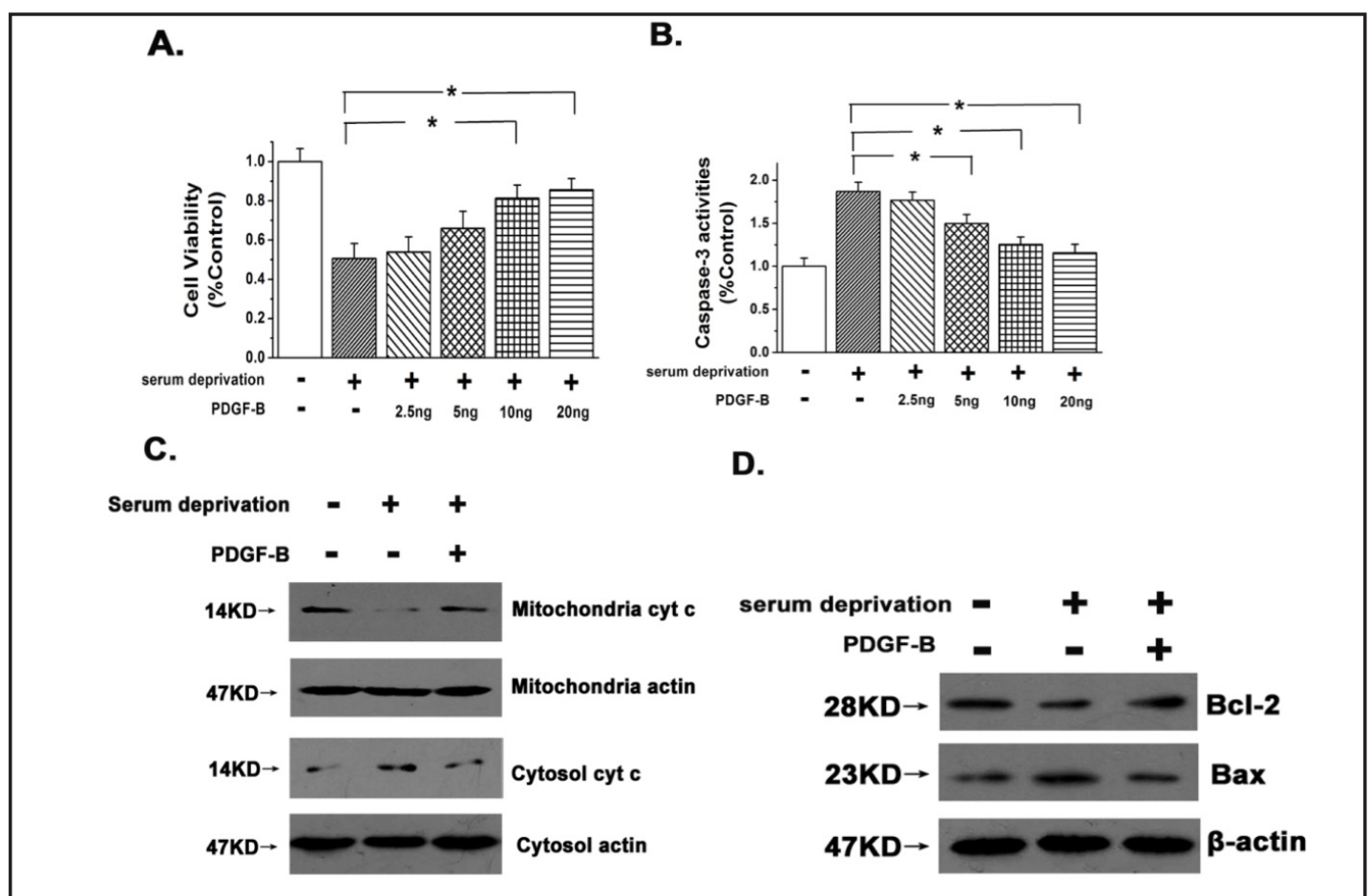

Fig. 3. PDGF-B inhibits PAECs apoptosis in a dose-dependent manner. A: PDGF-B improved cell viability at a concentration of $10 \mathrm{ng} / \mathrm{ml}$. B: The activation of caspase-3, which was induced by serum deprivation, was significantly inhibited by $10 \mathrm{ng} / \mathrm{ml}$ of PDGF-B. C: Serum deprivation caused the release of cytochrome $\mathrm{c}$ from the mitochondria to the cytosol, and this effect was reversed by PDGF-B treatment. D: PDGF-B inhibited the expression of Bax and induced the expression of Bcl-2 in starved PAECs. All values are denoted as means \pm SEMs from three or more independent batches of cells $\left({ }^{*} p<0.05\right)$.

Additionally, we examined the effects of PDGF-B on apoptosis in the setting of serum deprivation. As shown in Figure 3C, the mitochondrial cytochrome c level in PAECs was decreased, whereas the cytosolic cytochrome c levels were increased, in response to serum deprivation; however, PDGF-B mitigated the release of cytochrome c from the mitochondria to the cytosol in starved PAECs. Additionally, the results of western blot analysis revealed that an elevated expression level of Bax and a decreased expression level of Bcl-2 were detected in PAECs following serum deprivation, and these effects were reversed following the addition of PDGF-B (Fig. 3D, n=3, p<0.05). These results indicate that PDGF-B antagonizes intrinsic apoptosis.

PDGF-B enhances the phosphorylation of both Akt and Stat3 and promotes the nuclear translocation of Stat3

Both Akt and Stat3 play important roles in the survival and proliferation of many types of cells. Therefore, we next evaluated whether the activation and phosphorylation of these proteins were affected by PDGF-B in PAECs. As shown in Figures 4A and 4B, PDGF-B elicited the phosphorylation of both Akt (Ser473) and Stat3 (Tyr705) in PAECs. Moreover, the nuclear expression of Stat3 (activated Stat3) was significantly elevated in PAECs treated with PDGF-B, an effect that was accompanied by the decreased expression of cytoplasmic Stat3 (inactivated Stat3) (Fig. 4C, n=3, p<0.05). These results indicate that both Akt and Stat3 can be activated by PDGF-B in PAECs in the setting of serum deprivation.

AKT inhibition attenuates the increased phosphorylation of Stat3 elicited by PDGF-B in PAECs

The above results demonstrated that PDGF-B activated both the Akt and the Stat 3 signaling pathways, and previous reports have demonstrated the existence of cross-talk 
Fig. 4. PDGF-B enhances the phosphorylation of both Akt and Stat3 and promotes the nuclear translocation of Stat3. A: PDGF-B elicited the phosphorylation of Akt (Ser473) in PAECs. B: PDGF-B induced the phosphorylation of Stat3 (Tyr705). C: PDGF-B significantly elevated the nuclear expression of Stat3, which was accompanied by the decreased expression of cytoplasmic Stat3. All experiments were repeated with three or more independent batches of cells $(* \mathrm{p}<0.05)$.

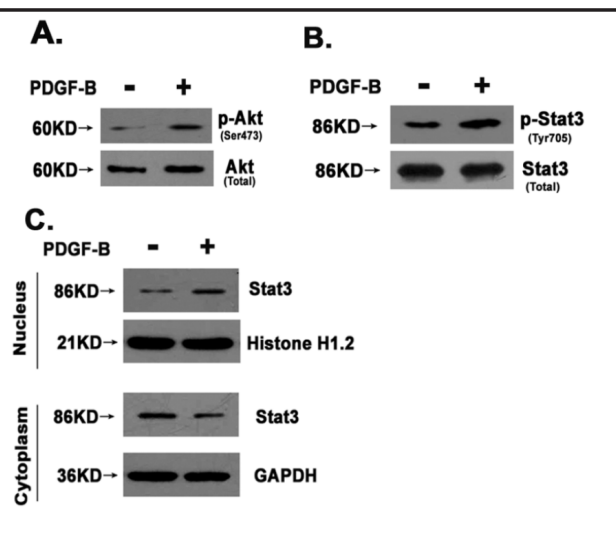

Fig. 5. The PI3K/ AKT pathway mediates activation of the Stat3 pathway in response to PDGF-B in PAECs, and the inhibitory effects of PDGF-B on apoptosis can be antagonized with a Stat3 inhibitor. A: LY294002 abolished the phosphorylation of both Akt and Stat3, whereas Stattic exerted no detectable effects on the phosphorylation of Akt. B: Blocking the Stat3 pathway attenuated the pro-
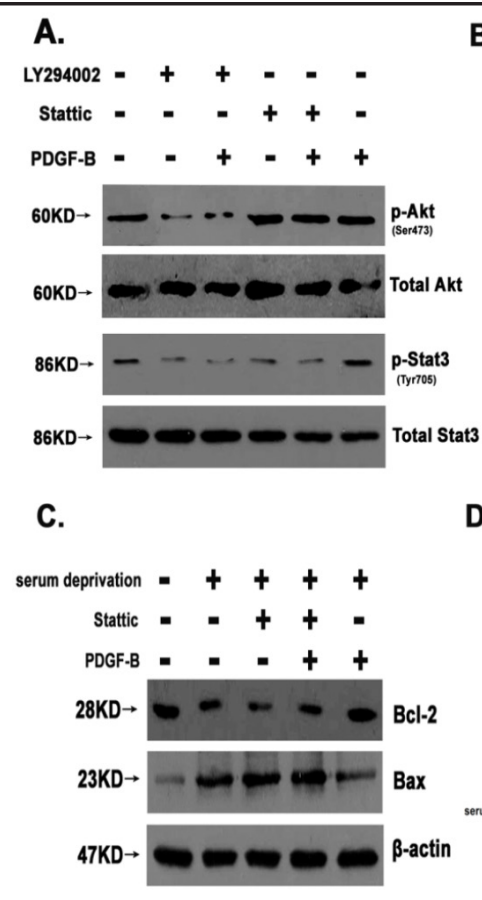

B.

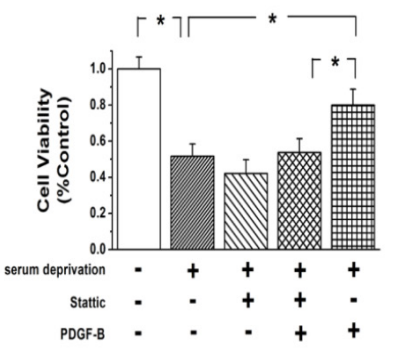

D.

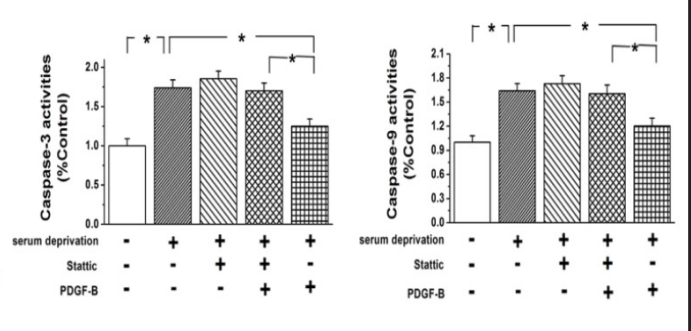

tective effects of PDGF-B on cell viability in PAECs. C: The increased expression of Bcl-2 and the decreased expression of Bax induced by PDGF-B were repressed by treatment with Stattic. D: PDGF-B inhibited the activation of both caspase-3 and caspase-9, and this effect was attenuated by treatment with a Stat3 inhibitor (Stattic).

between the JAK/Stat and PI3K/Akt pathways [24-26]. Therefore, LY294002 (an inhibitor of PI3K) and Stattic (a Stat3-specific inhibitor) were utilized for our research. As shown in Figure 5A, each inhibitor effectively prevented the phosphorylation of Akt or Stat3 respectively. Moreover, we found that the PI3K/Akt inhibitor (LY294002) not only abolished Akt activation but also precipitated a decrease in Stat3 phosphorylation. However, the Stat3specific inhibitor decreased only the phosphorylation of Stat3; there were no detectable effects on Akt phosphorylation (Fig. 5A, n=3, p<0.05). These results indicate that Akt likely serves as an upstream regulator of Stat3.

PDGF-B protects against the mitochondria-dependent apoptotic pathway via the Stat3 signaling pathway

We next targeted Stat3 with either Stattic (Stat3 inhibitor) or Stat3 siRNA to investigate the role of Stat3 signaling in the anti-apoptotic effects of PDGF-B under conditions of serum deprivation. We found that blockade of the Stat3 pathway attenuated the protective effects 


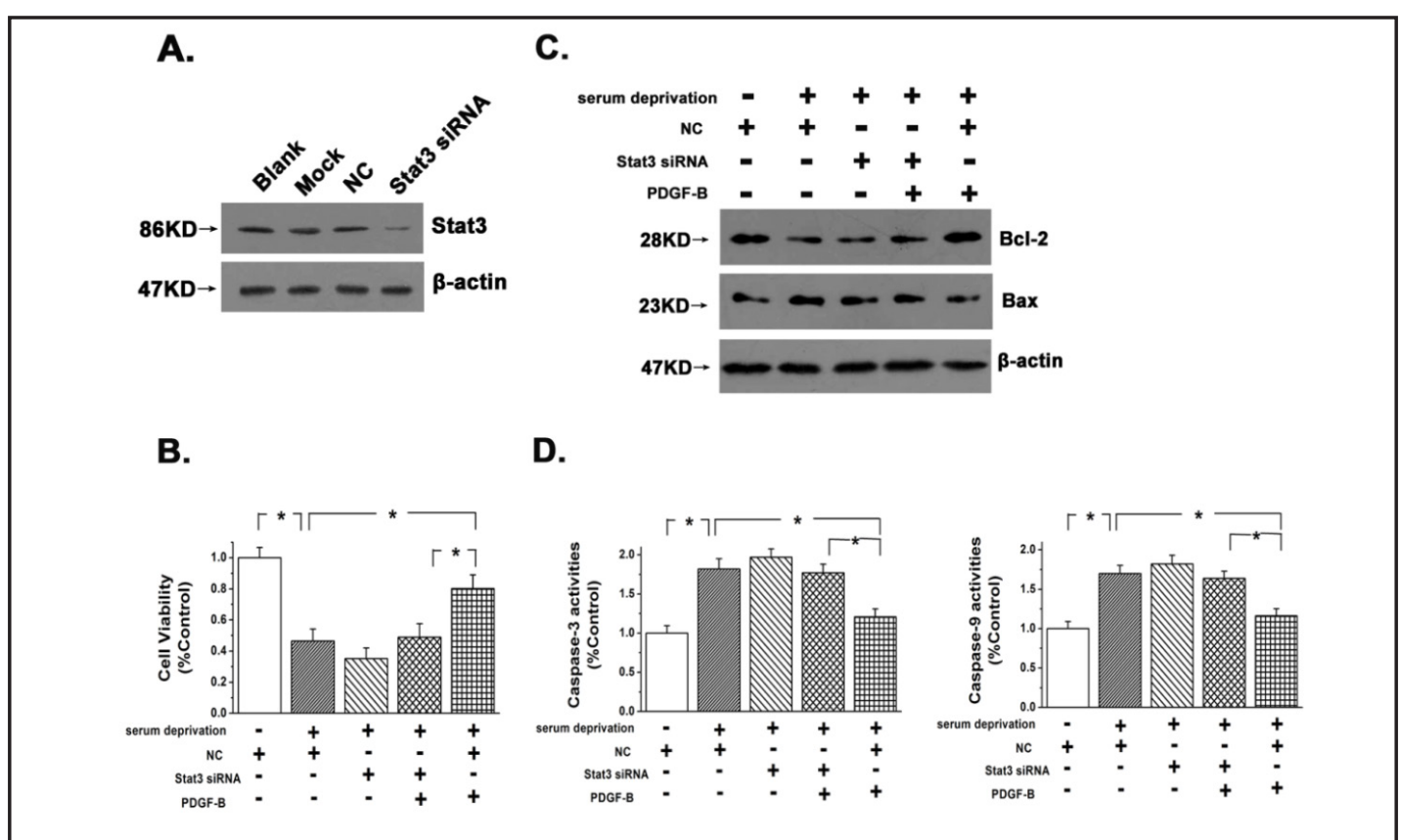

Fig. 6. The anti-apoptotic effects of PDGF-B can also be abolished by knocking down the expression of Stat3 with siRNA. A: The expression of Stat3 was knocked down with a specific Stat3 siRNA, as determined via western blotting. B: The protective effects of PDGF-B on cell viability were weakened following treatment with Stat3 siRNA. C: PDGF-B induced the expression of Bcl-2 and inhibited the expression of Bax in starved PAECs, and these effects of PDGF-B were abolished after knocking down Stat3 expression. D: PDGF-B depressed the activity of both caspase- 3 and caspase- 9 in a Stat3-dependent manner.

of PDGF-B on cell viability in PAECs and that the increased expression of Bcl-2 and decreased expression of Bax induced by PDGF-B were reversed by treatment with Stattic (Figs. 5B and $5 C, n=3, p<0.05)$. Specific siRNA designed to silence Stat3 gene expression was used to eliminate the possible nonspecific inhibition caused by the chemical inhibitor in PAECs, and western blotting was utilized to ensure the adequate siRNA-mediated knock down of Stat3 (Fig. 6A, n=3). The results showed that the anti-apoptotic effects of PDGF-B were also abolished by inhibition of Stat 3 with siRNA (Figs. 6B and 6C, $n=3, p<0.05$ ), which supported the findings shown in Figure 5. Furthermore, PDGF-B inhibited the activation of both caspase- 3 and caspase- 9 in a Stat3-dependent manner (Figs. 5D and 6D, n=3, $p<0.05$ ). These results indicate that PDGF-B protects against mitochondria-dependent pathway apoptosis via the Stat3 signaling pathway.

\section{Discussion}

It has been reported that hypoxia, which causes PAH, induces pulmonary vasoconstriction. Moreover, an important change that occurs in the intimal layer of the pulmonary vasculature is the overgrowth of PAECs and the development of plexiform lesions formed by PAECs during the progression of PAH, which obliterates the pulmonary arteries and results in luminal narrowing. However, the mechanisms underlying the effects of hypoxia on pulmonary vascular remodeling, particularly the changes in the vascular endothelium, are not well understood. In the present study, we provide new evidence that PDGF-B, which is induced by hypoxia, promotes the survival of starved PAECs and that the inhibitory effects exerted by PDGF-B on the mitochondria-dependent apoptotic pathway are mediated via the Akt/Stat3 pathway. These results therefore provide novel insights into the regulatory mechanism associated with the effects of PDGF-B on PAEC survival in the setting of hypoxia. 
A key finding of our study was that PDGF-B mediates the inhibitory effects exerted by hypoxia on PAECs. It is known that pulmonary vasoconstriction and pulmonary arterial remodeling are two basic pathological changes associated with the progression of pulmonary hypertension and that hypoxia plays an important role in both processes; indeed, the overgrowth of the pulmonary artery endothelium is caused by hypoxia and is responsible for pulmonary vascular remodeling $[4,27,28]$. However, information detailing how hypoxia advances the remodeling of the pulmonary arterial vasculature is scarce. Exploring the mechanism by which hypoxia regulates pulmonary arterial remodeling may help us to prevent the progression of PAH. PAEC overgrowth results primarily from disturbances in the balance between cell proliferation and apoptosis, as both the inhibition of apoptosis and the promotion of proliferation will enhance the growth of PAECs and result in both pulmonary arterial intimal hypertrophy and increased blood pressure [29, 30]. Our results indicate that hypoxia induces both the protein and mRNA expression of PDGF-B in PAECs. Moreover, the inhibitory effects of hypoxia on the apoptosis induced by serum deprivation were attenuated using inhibitors of the PDGF-B receptor in PAECs; thus, PDGF-B antagonizes intrinsic apoptosis in a concentration-dependent manner. These results provide novel insights that PDGF-B appears to be an important mediator in hypoxia-induced pulmonary vascular remodeling.

Accumulating data have shown that both the JAK/Stat pathway and the PI3K/Akt signaling pathway are classic pro-survival pathways in various types of cells [31-33]. Protein kinase B (Akt) is the central protein in the phosphatidylinositol-3 kinase (PI3K)/Akt signaling pathway, and the PI3K/Akt pathway is frequently over-activated during cell growth [34-36]. Stat3, an important transcription factor, is phosphorylated and subsequently translocates into the nucleus, where it binds to several target gene promoter sequences and exert its prosurvival effects. As we have established that both the JAK/Stat and the PI3K/Akt pathways are activated following stimulation by PDGF-B and that there is cross-talk between the JAK/ Stat and PI3K/Akt pathways [24-26], we sought to determine whether Stat3 or Akt serves as the upstream molecule in PAECs stimulated by PDGF-B. We observed that blocking the PI3K pathway with a specific inhibitor abolished the phosphorylation of both Akt and Stat3, whereas Akt phosphorylation was not affected following the inhibition of Stat3 in PAECs. These results indicate that Akt serves as an upstream regulator of Stat3 in the setting of PDGF-B treatment in PAECs.

Both Bcl-2 and Bax are located on the outer mitochondrial membrane and are involved in the regulation of mitochondrial stability; Bcl-2 is an anti-apoptotic protein, and Bax is a pro-apoptotic protein. The decreased ratio of Bcl-2 to Bax is often accompanied by a decline in mitochondrial membrane potential, which subsequently leads to the release of cytochrome c from the mitochondria to the cytoplasm, as well as the activation of both caspase- 3 and caspase-9 and the triggering of cell apoptosis [37-39]. In PAECs, we used serum deprivation as an apoptotic model and observed that the decrease in the ratio of Bcl-2/Bax induced by serum deprivation was inhibited by PDGF-B. In addition, PDGF-B blocked the release of cytochrome $\mathrm{c}$ from the mitochondria to the cytoplasm and reversed the activation of both caspase-3 and caspase- 9 following serum deprivation. However, each of the effects exerted by PDGF-B on cell apoptosis was inhibited following the blockade of the Stat3 pathway with either Stat3 siRNA or an inhibitor. Based on these results, we can conclude that PDGF-B protects against the mitochondria-dependent apoptotic pathway via the Akt/Stat3 signaling pathway in PAECs.

Although we demonstrated that PDGF-B enhances the phosphorylation of both Akt and Stat3 and activates the Akt/Stat3 pathway, whether other parallel signaling pathways are also regulated by PDGF-B to promote the survival of PAECs needs to be further determined. Additionally, relevant in vivo experiments should be performed in future studies to confirm the important role played by PDGF-B in pulmonary vascular remodeling.

In conclusion, the findings of the present study indicate that PDGF-B mediates the hypoxia-induced promotion of growth and survival of PAECs and also protects against the mitochondria-dependent apoptotic pathway via activation of the Akt/Stat3 pathway 
Li et al.: PDGF-B Promotes PAECs Survival via PI3K/Akt/Stat3

in PAECs. These results indicate that PDGF-B may play a role in the outgrowth of PAECs, which precedes pulmonary vascular remodeling. Additionally, these findings may help both clinicians and researchers develop novel therapies for the treatment of hypoxic pulmonary hypertension.

\section{Acknowledgement}

This work was supported by the Youth Science Foundation of Heilongjiang Province.

\section{Disclosure Statement}

None.

\section{References}

1 Shao D1, Park JE, Wort SJ: The role of endothelin-1 in the pathogenesis of pulmonary arterial hypertension. Pharmacol Res 2011;63:504-511.

2 Simonneau G, Robbins IM, Beghetti M, Channick RN, Delcroix M, Denton CP, Elliott CG, Gaine SP, Gladwin MT, Jing ZC, Krowka MJ, Langleben D, Nakanishi N, Souza R: Updated clinical classification of pulmonary hypertension. J Am Coll Cardiol 2009;54:S43-54.

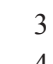
Haworth SG: Pulmonary endothelium in the perinatal period. Pharmacol Rep 2006;58:S153-64. Budhiraja R, Tuder RM, Hassoun PM: Endothelial dysfunction in pulmonary hypertension. Circulation 2004;109:159-165.

-5 Celermajer DS, Cullen S, Deanfield JE: Impairment of endothelium-dependent pulmonary artery relaxation in children with congenital heart disease and abnormal pulmonary hemodynamics. Circulation 1993;87:440-446.

6 Oechslin E, Kiowski W, Schindler R, Bernheim A, Julius B, Brunner-La Rocca HP: Systemic endothelial dysfunction in adults with cyanotic congenital heart disease. Circulation 2005;112:1106-1112.

7 Mukai Y, Rikitake Y, Shiojima I, Wolfrum S, Satoh M, Takeshita K, Hiroi Y, Salomone S, Kim HH, Benjamin LE, Walsh K, Liao JK: Decreased vascular lesion formation in mice with inducible endothelial-specific expression of protein kinase Akt. J Clin Invest 2006;116:334-343.

8 Monick MM, Hunninghake GW: Activation of second messenger pathways in alveolar macrophages by endotoxin. Eur Respir J 2002;20:210-222.

-9 Chow LQ, Eckhardt SG: Sunitinib: from rational design to clinical efficacy. J Clin Oncol 2007;25:884-896.

10 Faivre S, Demetri G, Sargent W, Raymond E: Molecular basis for sunitinib efficacy and future clinical development. Nat Rev Drug Discov 2007;6:734-745.

11 Darnell JE, Kerr IM, Stark GR: Jak-STAT pathways and transcriptional activation in response to IFNs and other extracellular signaling proteins. Science 1994;264:1415-1421.

12 Mertens C, Darnell JE: Snapshot: JAK-STAT signaling. Cell 2007;131:612.

13 Sehgal PB, Levy DE, Hirano T. (editors): Signal Transducers and Activators of Transcription (STATs): Activation and Biology. Dordrecht, The Netherlands: Kluwer Academic Press 2003, pp 1-746.

14 Sehgal PB: Plasma membrane rafts and chaperones in cytokine/STAT signaling. Acta Biochim Pol 2003;50:583-594.

15 Turkson J: STAT proteins as novel targets for cancer drug discovery. Expert Opin Ther Targets 2004;8:409422.

16 Myllarniemi M, Calderon L, Lemstrom K, Buchdunger E, Hayry P: Inhibition of platelet-derived growth factor receptor tyrosine kinase inhibits vascular smooth muscle cell migration and proliferation. FASEB J 1997;11:1119-1126.

17 Sano H, Sudo T, Yokode M, Murayama T, Kataoka H, Takakura N, Nishikawa S, Nishikawa SI, Kita T: Functional blockade of platelet-derived growth factor receptor-beta but not of receptor-alpha prevents vascular smooth muscle cell accumulation in fibrous cap lesions in apolipoprotein E-deficient mice. Circulation 2001;103:2955-2960. 


\begin{tabular}{|c|c|c|}
\hline Cellular F & Cell Physiol Biochem 2015;35:441-451 & \\
\hline and Biochemistry & $\begin{array}{l}\text { DOI: 10.1159/000369709 } \\
\text { Publisned onilne. January } 15,2015\end{array}$ & $\begin{array}{l}\text { O } 2015 \text { S. Karger AG, Basel } \\
\text { www.karger.com/cpb }\end{array}$ \\
\hline
\end{tabular}

18 Seifert RA, Hart CE, Phillips PE, Forstrom JW, Ross R, Murray MJ, Bowen-Pope DF: Two different subunits associate to create isoform-specific platelet-derived growth factor receptors. J Biol Chem 1989;264:87718778.

19 Kawai-Kowase K, Owens GK: Multiple repressor pathways contribute to phenotypic switching of vascular smooth muscle cells. Am J Physiol Cell Physiol 2007;292:C59-C69.

-20 Ogawa A, Nakamura K, Matsubara H, Fujio H, Ikeda T, Kobayashi K, Miyazaki I, Asanuma M, Miyaji K, Miura D, Kusano KF, Date H, Ohe T: Prednisolone inhibits proliferation of cultured pulmonary artery smooth muscle cells of patients with idiopathic pulmonary arterial hypertension. Circulation 2005;112:1806-1812.

21 Medhora M1, Chen Y, Gruenloh S, Harland D, Bodiga S, Zielonka J, Gebremedhin D, Gao Y, Falck JR, Anjaiah S, Jacobs ER: 20-HETE increases superoxide production and activates NAPDH oxidase in pulmonary artery endothelial cells. Am J Physiol Lung Cell Mol Physiol 2008;294:L902-911.

22 Wang Z, Tang X, Li Y, Leu C, Guo L, Zheng X, Zhu D: 20-Hydroxyeicosatetraenoic acid inhibits the apoptotic responses in pulmonary artery smooth muscle cells. Eur J Pharmacol 2008;588:9-17.

23 Ma J, Zhang L, Li S, Liu S, Ma C, Li W, Falck JR, Manthati VL, Reddy DS, Medhora M, Jacobs ER, Zhu D: 8,9-Epoxyeicosatrienoic acid analog protects pulmonary artery smooth muscle cells from apoptosis via ROCK pathway. Exp Cell Res 2010;316:2340-2353.

24 Mao W, Iwai C, Liu J, Sheu SS, Fu M, Liang CS: Darbepoetin alfa exerts a cardioprotective effect in autoimmune cardiomyopathy via reduction of ER stress and activation of the PI3K/Akt and STAT3 pathways. J Mol Cell Cardiol 2008;45:250-260.

25 Zhang X, Shan P, Alam J, Fu XY, Lee PJ: Carbon monoxide differentially modulates STAT1 and STAT3 and inhibits apoptosis via a PI3K/Akt and p38 kinase dependent STAT3 pathway during anoxiareoxygenation injury. J Biol Chem 2005;280:8714-8721.

26 Ponnusamy M, Pang M, Annamaraju PK, Zhang Z, Gong R, Chin YE, Zhuang S: Transglutaminase-1 protects renal epithelial cells from hydrogen peroxide-induced apoptosis through activation of STAT3 and AKT signaling pathways. Am J Physiol Renal Physiol 2009;297:F1361-1370.

27 Farkas L, Gauldie J, Voelkel NF, Kolb M: Pulmonary hypertension and idiopathic pulmonary fibrosis: a tale of angiogenesis, apoptosis, and growth factors. Am J Respir Cell Mol Biol 2011;45:1-15.

-28 Taraseviciene-Stewart L, Kasahara Y, Alger L, Hirth P, Mc Mahon G, Waltenberger J, Voelkel NF, Tuder RM: Inhibition of the VEGF receptor 2 combined with chronic hypoxia causes cell death-dependent pulmonary endothelial cell proliferation and severe pulmonary hypertension. FASEB J 2001;15:427-438.

29 Xu X, Zhang XA, Wang DW: The roles of CYP450 epoxygenases and metabolites, epoxyeicosatrienoic acids, in cardiovascular and malignant diseases. Adv Drug Deliv Rev 2011;63:597-609.

- 30 Orlandi A, Francesconi A, Cocchia D, Corsini A, Spagnoli LG: Phenotypic heterogeneity influences apoptotic susceptibility to retinoic acid and cis-platinum of rat arterial smooth muscle cells in vitro: Implications for the evolution of experimental intimal thickening. Arterioscler Thromb Vasc Biol 2001;21:1118-1123.

- 31 Sarker D, Reid AH, Yap TA, de Bono JS: Targeting the PI3K/AKT pathway for the treatment of prostate cancer. Clin Cancer Res 2009;15:4799-805.

-32 Huang S: Regulation of metastases by signal transducer and activator of transcription 3 signaling pathway: clinical implications. Clin Cancer Res 2007;13:1362-1366.

-33 Goswami A, Ranganathan P, Rangnekar VM: The phosphoinositide 3-kinase/Akt1/Par-4 axis: a cancerselective therapeutic target. Cancer Res 2006;66:2889-2892.

-34 Datta SR, Brunet A, Greenberg ME: Cellular survival: a play in three Akts. Genes 1999;13:2905-2927.

- 35 Katso R, Okkenhaug K, Ahmadi K, White S, Timms J, Waterfield MD: Cellular function of phosphoinositide 3-kinases: implications for development, homeostasis, and cancer. Annu Rev Cell Dev Biol 2001;17:615675.

-36 Luo J, Manning BD, Cantley LC: Targeting the PI3K-Akt pathway in human cancer: rationale and promise. Cancer Cell 2003;4:257-262.

37 Lei X, Chen Y, Du G, Yu W, Wang X, Qu H, Xia B, He H, Mao J, Zong W, Liao X, Mehrpour M, Hao X, Chen Q: Gossypol induces Bax/Bak-independent activation of apoptosis and cytochrome c release via a conformational change in Bcl-2. FASEB J 2006;20:2147-2149.

- 38 Ding J, Zhang Z, Roberts GJ, Falcone M, Miao Y, Shao Y, Zhang XC, Andrews DW, Lin J: Bcl-2 and Bax interact via the BH1-3 groove-BH3 motif interface and a novel interface involving the BH4 motif. J Biol Chem 2010;285:28749-2863.

-39 Childs AC, Phaneuf SL, Dirks AJ, Phillips T, Leeuwenburgh C: Doxorubicin treatment in vivo causes cytochrome $\mathrm{C}$ release and cardiomyocyte apoptosis, as well as increased mitochondrial efficiency, superoxide dismutase activity, and Bcl-2:Bax ratio. Cancer Res 2002;62:4592-4598. 